\title{
The Kinematics and Kinetics Analysis of the Lower Extremity in the Landing Phase of a Stop-jump Task
}

\author{
L. Yin ${ }^{1,2}$, D. Sun ${ }^{1}$, Q.C. Mei ${ }^{1}$, Y.D. Gu ${ }^{1, *}$, J.S. Baker ${ }^{2}$ and N. Feng ${ }^{3}$ \\ ${ }^{1}$ Human Movement Research Center, Faculty of Sports Science, Ningbo University, Zhejiang 315211, China \\ ${ }^{2}$ School of Science and Sport, University of the West of Scotland, Hamilton, ML3 OJB, Scotland \\ ${ }^{3}$ Rehabilitation Center, Ningbo Ninth Hospital, Zhejiang 315020, China
}

\begin{abstract}
Large number of studies showed that landing with great impact forces may be a risk factor for knee injuries. The purpose of this study was to illustrate the different landing loads to lower extremity of both genders and examine the relationships among selected lower extremity kinematics and kinetics during the landing of a stop-jump task. A total of 35 male and 35 female healthy subjects were recruited in this study. Each subject executed five experiment actions. Lower extremity kinematics and kinetics were synchronously acquired. The comparison of lower extremity kinematics for different genders showed significant difference. The knee and hip maximum flexion angle, peak ground reaction force and peak knee extension moment have significantly decreased during the landing of the stop-jump task among the female subjects. The hip flexion angle at the initial foot contact phase showed significant correlation with peak ground reaction force during landing of the stop-jump task $(\mathrm{r}=-0.927, \mathrm{P}<0.001)$. The knee flexion angle at the initial foot contact phase had significant correlation with peak ground reaction force and vertical ground reaction forces during landing of the stop-jump task ( $\mathrm{r}=-0.908, \mathrm{P}<0.001 ; \mathrm{r}=0.812, \mathrm{P}=0.002)$. A large hip and knee flexion angles at the initial foot contact with the ground did not necessarily reduce the impact force during landing, but active hip and knee flexion motions did. The hip and knee flexion motion of landing was an important technical factor that affects anterior cruciate ligament (ACL) loading during the landing of the stop-jump task.
\end{abstract}

Keywords: Anterior cruciate ligament, correlation, extension, flexion, landing, stop-jump.

\section{INTRODUCTION}

Landing is one of the most frequent actions in the exercise movement. A good landing pattern is not only a sign of competitively technical requirement, but also effective in preventing lower limbs injuries. Large amount of previous studies showed that landing with great impact forces may be a risk factor to knee injuries, particularly the anterior cruciate ligament tear [1-5]. Many researches have attempted to analyze the biomechanical mechanism of landing, in order to reduce the impact force during landing and the loading to knee joint [6]. Devita [1] showed that the increase of knee flexion angles could effectively reduce the ground reaction force. Chappell [3] found that comparing to male amateur athletes, the peak value of shear force to the front side of tibia and peak flexion and extension torque of knee to female amateur athletes has increased averagely, but knee flexion angle has decreased during the landing of a stop-jump task. A further study found that under fatigue situation the knee flexion angle of male and female amateur athletes decreased while the initial contact with the ground. But when they have the landing of stop-jump, shear force to the proximal tibia front side increased averagely. These results indicate that

\footnotetext{
*Address correspondence to the author at the Human Movement Research Center, Faculty of Sports Science, Ningbo University, Zhejiang 315211, China; E-mail: guyaodongnb@aliyun.com
}

when the foot initially contact the ground the increasing of knee flexion angle may reduce the impact force during landing and loading to knee joint. However, Yu [6] reported that the knee flexion angle increasing $5^{\circ}$ at foot initial contacting with the ground not producing significant effects on ground reaction force during the landing of a stop-jump. Decker [5] also reported that the kinematics of hip, knee, ankle between the different genders has produced significant changes during the landing of vertical jump, but kinetics of lower limb has not caused any significant difference between different genders.

There are conflicting results in the biomechanical studies of the landing, and mainly because people lack cognitive interrelation of kinematic parameters and kinetic parameter during landing. The previous studies concerning kinematics and kinetics of lower limbs only compared the differences between kinetic and kinematic parameters of the lower limbs, ignoring the accurate analysis of the relationship between the kinematics and kinetic parameters of lower limbs [1-5]. If the relationship of kinetic and kinematic parameters during landing couldn't be understood, it would inhibit the effective use of biomechanical parameters of the lower limbs for injury prevention.

The purpose of this study was to examine the relationship between the selected lower extremity kinematics and kinetics 
during the landing of a stop-jump task between male and female. The stop-jump movement frequently existed in many sports, such as basketball, volleyball, rhythmic gymnastics, aerobics, etc [7, 8]. It was hypothesized that there existed significant difference between kinetic and kinematic parameters of different genders while landing, and the angles and angular velocity of knee and hip showed a significant correlation with ground reaction force and anterior-posterior friction in the landing phase.

\section{MATERIALS AND METHODS}

\subsection{Subjects}

Seventy healthy men and women volunteered for the research, with 35 males and 35 females participating in the test (Table 1). All subjects are students of Ningbo University. Before experiment, written were informed consent was obtained. None of these subjects had any previous injury history to the lower extremity. All subjects had engaged in basketball training or aerobics training, and performed vigorous exercise twice a week, two hours at a time.

\subsection{Procedures}

The tests were performed in the Sports Biomechanics Laboratory of Ningbo University. Technical actions performed are the Stop-Jump. Specific action requirements are slow run-up, single foot step and immediate stop, and feet vertical jump-up (Fig. 1). These actions appear frequently in basketball and volleyball, such as pulling-up jumpers in basketball, spiking in volleyball, and so on.

Before the experimental data collection, each subject conducted warming-up for about 10 minutes, and then professional basketball players demonstrated the stop-jump movement, including the interpretation of action points.
After the guidance of professional athletes, each subject was familiar with the action, in order to avoid being affected by the accuracy of the experimental data because of action unfamiliarity. During the experiment, all subjects were unaware of the content of the research.

The acquisition of three-dimensional kinematic and kinetic data was conducted synchronously, separately collecting data by the 8 cameras $(250 \mathrm{~Hz})$ of motion capture system Vicon (Oxford Metrics Limited UK) and a force platform (Kistler, Switzerland) $(1000 \mathrm{~Hz})$. Vicon system controls experimental error in the following $0.5 \mathrm{~mm}$. Before the experiment, it was required to attach reflective markers to the lower limbs of subjects, and affixed reflective markers were divided into two groups. Markers of one group were attached to the pelvic area (4): Left and right anterior superior iliac spine, left and right posterior superior iliac spine. Markers of another group were attached to the lower limbs (12): left and right thigh, left and right knee, left and right shin, left and right lateral ankle joint, left and right toe, left and right heel. In order to distinguish left and right side, the position of reflective points attached to the right thigh was lower than the left thigh. After attaching, the subjects stood still and upright in the center position of the testing area, the direction of foot longitudinal arch and $\mathrm{Y}$-axis coordinate system was consistent, then static calibration. The purpose was to determine the location of joint through various parts of sign points. After static calibration, subjects stood still on the force platform to entry weight data. Then each subject ran on the runway with length of 10 meters. Immediate stop and landing took place at the force platform. Each subject completed five trials. In order to avoid the influence of fatigue during the experiment to the accuracy of the experiment, subjects rested for two minutes after completing one experiment action. When players completed experimental action, reflective markers would also be removed. Vicon system can capture the light reflected off the signs point, record moving track of signs point, the same

Table 1. Subjects Information.

\begin{tabular}{|c|c|c|c|c|}
\hline Gender (N) & Age (yr) & Height (m) & Weight $(\mathbf{k g})$ & BMI $\left(\mathbf{k g} / \mathbf{m}^{2}\right)$ \\
\hline \hline Male (35) & $21.7(2.5)$ & $1.74(0.12)$ & $70.3(8.4)$ & $23.22(0.42)$ \\
\hline Female (35) & $21.3(1.9)$ & $1.63(0.07)$ & $54.9(7.1)$ & $20.55(0.9)$ \\
\hline
\end{tabular}

Note: Mean (standard deviation, SD)
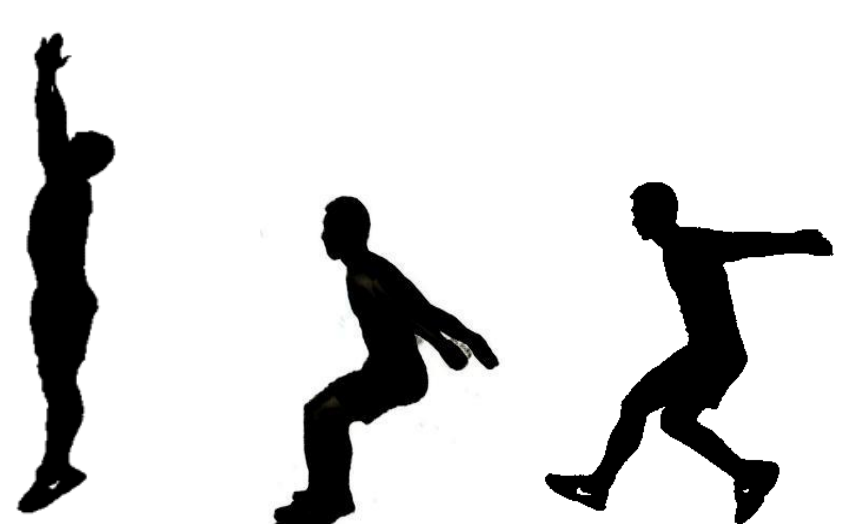

Fig. (1). The experiment movement from right to left: Single foot step, immediate stop, feet off-ground Changes of the frictional force in the longitudinal direction 
experiment recorded the action. Kinematics and kinetics data were collected through the low-pass filtering device were respectively cut off frequency of $20 \mathrm{~Hz}$ and $100 \mathrm{~Hz}$ data.

This research defined an action cycle as landing on the ground to jump off the ground. The moment of foot initial contact to the ground is the time when force value is greater than zero in the first frame data appears from the force platform. The moment of the jump-up corresponding to the time that force value from the force platform decreases approximately zero. The main essence of the action is divided into two stages, namely: single foot step and immediate stop stage, feet vertical take-off stage. Action process of single foot step and immediate stop stage is from the first touchdown of the foot to the knee flexion angle reaches the maximum. Action process of feet vertical takeoff stage is from the maximum knee flexion to jump-up. The biomechanical parameters extraction in two stages of this research include: (1) Joint angle and angular velocity of hip and knee during the initial touchdown of feet. (2) Maximum flexion angle of the hip and knee. (3) Peak of ground reaction force in the horizontal plane on single foot step and immediate stop stage and peak of ground reaction force in the vertical plane on feet vertical taking-off stage. (4) Flexion moment of knee on single foot step and immediate stop stage.

\subsection{Statistical Analysis}

The research selected the dominant leg (the leg on the step) as the research object. Joint angle and angular velocity of hip joint and knee during the initial touchdown of foot, maximum flexion of the hip and knee, peak of ground reaction force in the horizontal plane on single foot step and immediate stop stage and peak of ground reaction force in the vertical plane on feet vertical take-off stage, flexion moment of knee on single foot step and immediate stop stage. Differences of those data between different genders were in statistical analysis using SPSS version 17.0 software. A total of nine statistical analyses were performed. The analysis method included was independent samples t-test. Homogeneity of variance test was taken for comparisons of statistical data before t-test. The definition of homogeneity of variance is $\mathrm{P}>0.05$ (when the homogeneity of variance does not exist), $\mathrm{P}<0.05$ (when the homogeneity of variance exist), then the next t-test statistics could be obtained. Also in the ttest, $\mathrm{P}>0.05$ not statistically significant difference, whereas $\mathrm{P}<0.05$ is the statistically significant difference.

The Pearson correlation coefficient test was used to analyze correlation between the kinematics and kinetic parameters of lower limbs and the ground reaction force of horizontal and vertical directions. Lower extremity kinematics and kinetic parameters include: Hip and knee flexion angle and angular velocity when the feet initially touched the ground. Maximum hip and knee flexion angle was in the landing stage. Knee flexion angle when the peak of proximal tibia front side shear force appeared. Meanwhile, the correlation among the ground reaction force, peak of knee flexion and extension moment were examined. R-value described the degree of strength of the linear correlation between two variables. The definition of $|r| \geq 0.65$ means that it has correlation, $|r| \geq 0.8$ means that it has significant correlation. $\mathrm{P}$ value is to check whether a correlation exists between variables in the entire samples. The definition of $\mathrm{P}<0.05$ is the presence of entire samples which has a consistent correlation.

\section{RESULTS}

The knee and hip flexion angle significantly decreased during the landing of the stop-jump task with the female subjects. The maximum flexion angle of knee and hip was in the landing stage. The peak anterior-posterior ground reaction force and peak knee extension moment was significantly decreased during the landing of the stop-jump task $(P \leq 0.03)$ (Fig. 2, Table 2). At the same time, compared with male subjects, the knee flexion angular velocity at the initial foot contact with the ground and the peak moment of ground reaction force also reduced in female subjects, but there was no significant $(P \geq 0.09)$ (Table 2).

The hip flexion angle and peak anterior-posterior ground reaction force showed a significant correlation during the landing of the stop-jump task ( $\mathrm{r}=-0.927, P<0.001)$ (Table 3), showed no correlation with peak vertical ground reaction forces $(\mathrm{r}=0.209, P=0.514)$ (Table 3). Hip flexion angular velocity showed no correlation with peak posterior ground reaction force and peak vertical ground reaction forces at the initial foot contact with the ground $(\mathrm{r}=0.251, P=0.458$; $\mathrm{r}=-$ $0.211, P=0.534$ ) (Table 3). The maximum angular velocity of hip flexion showed correlation with peak posterior ground reaction force and peak vertical ground reaction forces during the landing of the stop-jump task $(\mathrm{r}=-0.743, P=0.009$; $\mathrm{r}=0.618, P=0.043$ ) (Table 3). The knee flexion angle at the initial foot contact had a significant correlation with peak posterior ground reaction force and vertical ground reaction forces during landing of the stop-jump task ( $\mathrm{r}=-0.908$, $P<0.001 ; \mathrm{r}=0.812, P=0.002$ ) (Table 3). The knee flexion angular velocity showed no correlation with peak anteriorposterior ground reaction force at the initial foot contact with the ground $(\mathrm{r}=-0.365, P=0.269)$ (Table 3$)$, showed no correlation with peak vertical ground reaction forces $(\mathrm{r}=0.596, P=0.053)$ (Table 3). The maximum knee flexion showed no correlation with the peak anterior-posterior ground reaction force and vertical ground reaction forces during landing of the stop-jump task $(\mathrm{r}=-0.001, P=0.997$; $\mathrm{r}=-$ $0.210, P=0.536$ ) (Table 3).

\section{DISCUSSION}

This study found that male and female subjects have significant difference in kinematics and kinetics of the lower extremities while performing a stop-jump and landing movement. Female subjects in this study showed a reduction of knee flexion angle in the initial contact of the ground, and this is consistent with previous findings of female landing with greater knee extension at ground contact [2, 3, 5]. Compared with male subjects, female subjects showed a significant decrease of longitudinal friction force and knee flexion moment before and after the immediate landing stage. This was thought to be a different landing strategy between female and male, as Decker et al [5] had put it that the pre-planned muscular strategy to adjust lower limb in a position of lower magnitude of peak loading. The ground reaction force (GRF) did not show significant changes, with 
female subjects showing a similar peak ground reaction force with male participants in the landing phase. In the initial landing stage, knee joint flexion angle decreased, which was consistent with the study of Devita and Skelly [1] about the increase of landing impact. This could be explained as the weak muscular function in the landing shock absorption strategy, and this gender-related technique might lead female to a higher risk of knee anterior cruciate ligament (ACL) injury $[2,5]$. Contrarily, male participants with greater knee and hip flexion angles tended to transfer the impact or collapse energy back up to large and strong lower extremity muscles to absorb these shocks [5, 6, 8]. In this study, as both group participants had to be compared to analyze the different landing loads characteristics, the body weight difference between male and female participants was normalization by individual BMI range group (in the normal BMI range), excluding the over-weight effect on the ground reaction forces [5, 16-19].

As it has been hypothesized, that the results of this study showed that the kinematic of hip and knee in the early falling stage affected the landing kinetics of lower limb in the immediate stop and landing stage. The result showed that female subjects could produce smaller flexion angles of hip and knee in the landing phase. Further analysis on the flexion angles of hip and knee in the early landing phase was significantly correlated with the longitudinal friction force and the vertical ground reaction force (GRF). But flexion angular velocity of hip and knee did not show any correlation. To summarize these results, the angle of hip and knee in the initial landing phase significantly affected the

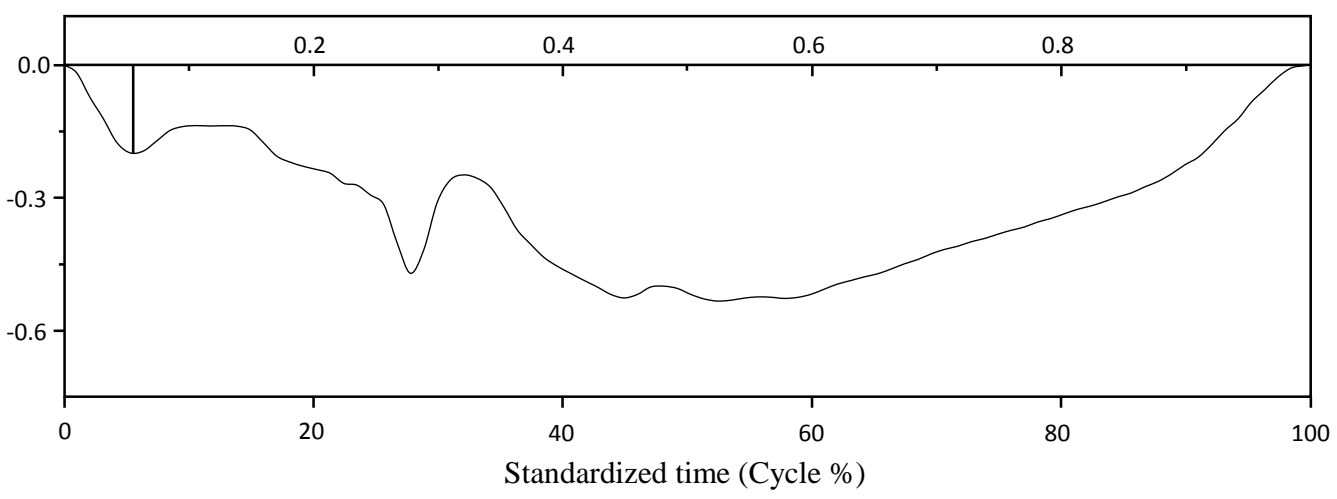

The change of ground reaction force (GRF) in a vertical direction (BW, bod weight)

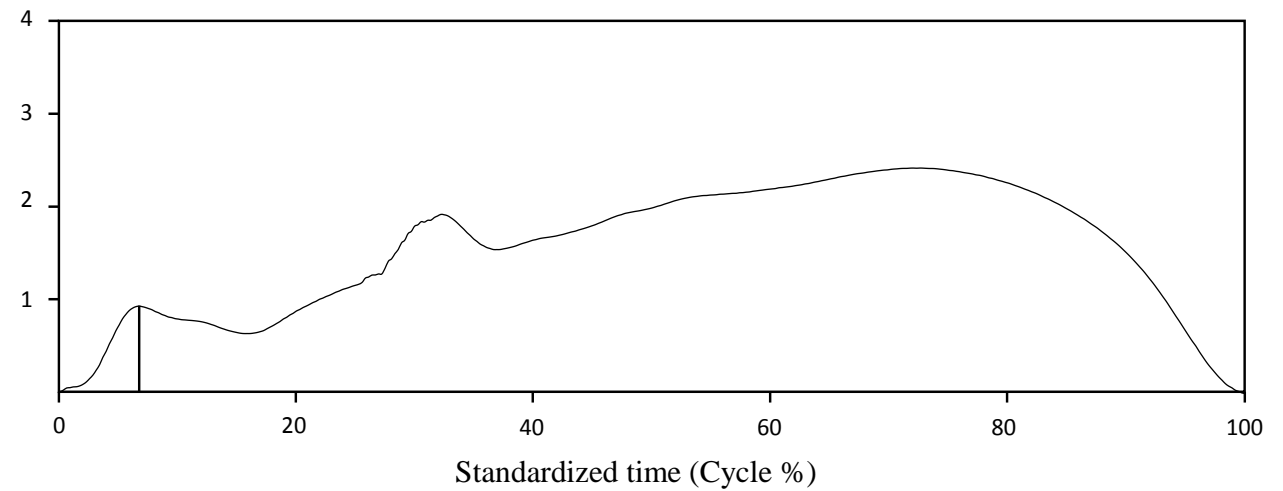

The change of knee flexion/extension torque (BW \& BH, body weight \& body height)

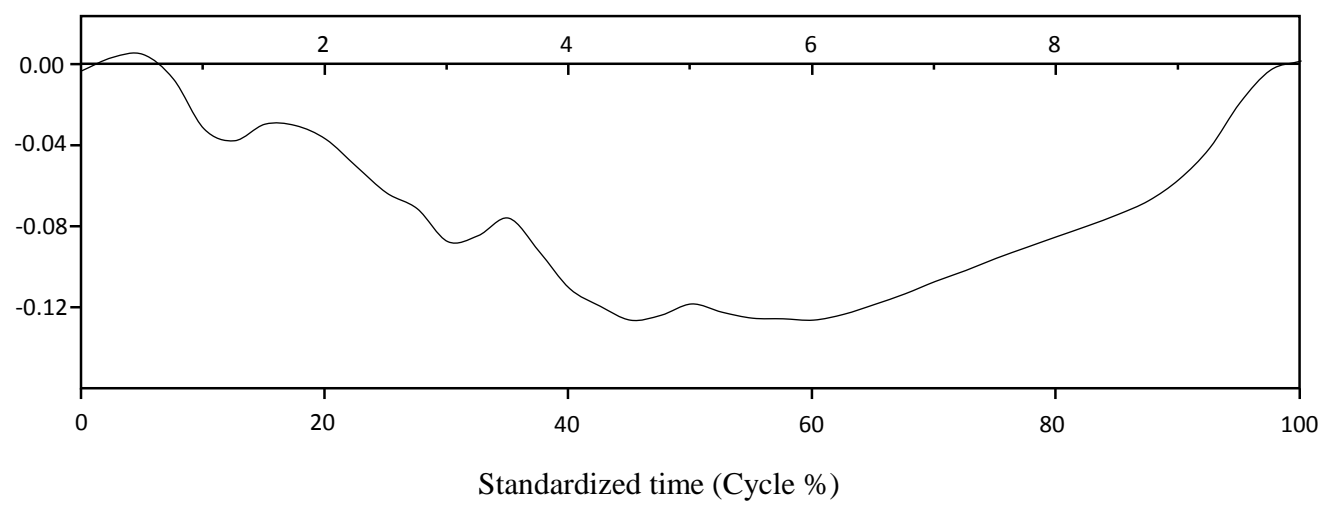

Fig. (2). Changes in the longitudinal direction of the frictional force, a vertical direction of ground reaction force and knee flexion / extension torque in the landing stage. 
Table 2. The mean (SD, standard deviation) value of lower extremity kinematics and kinetics during the landing of the stop-jump task between different genders.

\begin{tabular}{|c|c|c|c|}
\hline Variable & Male & Female & $\boldsymbol{P}$ \\
\hline Knee flexion at the initial contact of the ground $\left(^{\circ}\right)$ & $31.45(3.41)$ & $17.80(1.96)$ & $<0.001$ \\
\hline The maximum knee flexion during the landing of the stop-jump task $\left({ }^{\circ}\right)$ & $44.03(3.10)$ & $34.57(5.05)$ & 0.003 \\
\hline Hip flexion at the initial contact of the ground $\left({ }^{\circ}\right)$ & $49.26(1.67)$ & $28.55(0.91)$ & $<0.001$ \\
\hline The maximum hip flexion during the landing of the stop-jump task $\left(^{\circ}\right)$ & $60.47(2.06)$ & $39.95(1.94)$ & $<0.001$ \\
\hline Peak longitudinal GRF during the landing of the stop-jump task (BW) & $-0.63(0.03)$ & $-0.37(0.05)$ & $<0.001$ \\
\hline Peak GRF during landing of the stop-jump task (BW) & $3.36(0.05)$ & $2.99(0.43)$ & 0.09 \\
\hline
\end{tabular}

Table 3. The correlation coefficient between kinematic parameters of the hip and knee, peak longitudinal frictional force and vertical GRF during landing of the stop-jump task ( $p$ value).

\begin{tabular}{|l|c|c|}
\hline \multicolumn{1}{|c|}{ Kinematic parameters } & Peak longitudinal frictional force & Peak vertical GRF \\
\hline \hline Hip flexion at the initial foot contact of the ground & $-0.927(<0.001)$ & $0.209(0.514)$ \\
\hline Hip flexion angular velocity at the initial contact of the ground & $0.251(0.458)$ & $-2.11(0.534)$ \\
\hline The maximum hip flexion during the landing of the stop-jump task & $-0.743(0.009)$ & $0.618(0.043)$ \\
\hline Knee flexion at the initial contact of the ground & $-0.908(<0.001)$ & $0.812(0.002)$ \\
\hline Knee flexion angular velocity at the initial contact of the ground & $-0.365(0.269)$ & $0.596(0.053)$ \\
\hline The maximum knee flexion during landing of the stop-jump task & $-0.001(0.997)$ & $-0.210(0.536)$ \\
\hline
\end{tabular}

peak ground reaction force (GRF) and the peak longitudinal frictional force, but showed no effect on angular velocity of the hip and knee. The results showed that the range of motion (ROM) of hip and knee replaced the whole body function to alleviate the impact in the landing phase and showed an obvious effect. These results indicated that the activity and work from hip and knee joint instead of body function had a significant impact on the impact force in the landing stage. But the positive activity of hip and knee joint could reduce the impact $[6,17]$.

The movements of hip and knee joints play important roles in the change of the ground impact [5, 20-22]. The results of this study show the hip flexion angle have a greater influence on the longitudinal frictional force than the effect of knee's flexion angle, which is different with the male knee's extension work [5]. On the other hand, the influence of knee flexion angle on the vertical ground reaction force is greater than the influence of hip flexion angle in the early landing phase. The angles of lower limb joints bear loading in a specific joint structure. For instance, knee angle will affect the load to the anterior cruciate ligament (ACL). Fleming et al. [9] and Heijne et al. [10] showed that in the training of climbing stairs and blocking dynamics, the load of anterior cruciate ligament (ACL) decreased as the knee flexion angle increasing. Nunley [11] showed that axis rotational angle of the patella and tibia increased with the increasing of knee flexion angles. These findings suggested that as other conditions remain unchanged, the smaller angle of the knee flexion joint can lead to the larger load to anterior cruciate ligament (ACL). DeMorat [12] et al. reported that when the knee flexion angle reaches $20^{\circ}$, quadriceps muscle needs to generate the strength of $4500 \mathrm{~N}$. These results showed that the increased knee flexion angle could effectively reduce the non-direct contact damage to anterior cruciate ligament (ACL).

The biomechanical parameters of lower extremity could significantly affect the loading of anterior cruciate ligament (ACL). Previous studies also mentioned the knee flexion angle could significantly affect the loading to the anterior cruciate ligament (ACL). Some recent studies showed that the kinetic parameters of the knee in the sagittal plane could also effectively influence the impact loading to the knee. Fleming et al. [13] measured the tension of anterior cruciate ligament (ACL) while the knee conducting flexion and extension. They found that the knee flexion torque was significant correlated with anterior cruciate ligament (ACL) tension. Shelburne et al. [23] analyzed the load of the anterior cruciate ligament (ACL) in the walking gait. The loading are evaluated by the model of skeletal muscle. Their results showed that when the loading to the anterior cruciate ligament (ACL) of the knee joint reached the maximum, the muscle strength of quadriceps reached the maximum, and the 
knee flexion angle reached the minimum. Cerulli et al. [14] and Lamontagne et al. [15] studied the loading of anterior cruciate ligament (ACL) in the landing stage of small jump. Their study found that the increase of anterior cruciate ligament (ACL) tension occurred before the foot touchdown. When the knee joint flexion angle becoming the minimum, anterior cruciate ligament (ACL) tension and ground reaction forces together achieve the maximum. The results indicated that the lower limb kinetic parameters essentially occur at the same time, the non-contact of anterior cruciate ligament (ACL) plays an important role in the mechanism of injury in the analysis of biomechanics in the sagittal plane of lower extremity.

This study analyzed the different landing loads characteristics between male and female and biomechanical mechanisms of sagittal plane in the landing phase. As discussed, the loading of anterior cruciate ligament (ACL) is affected not only in the sagittal plane, but also in the coronal and horizontal plane. The future study should be related to other biomechanical parameters, such as adduction and abduction in the coronal plane and internal rotation and lateral rotation in the horizontal plane of the knee. This study only selected the stop-jump action. And this movement is one of the factors leading to the noncontact anterior cruciate ligament (ACL) injury in the form of the main action. But there are many forms of other actions that could result in non-contact anterior cruciate ligament (ACL) injury. Future research shall investigate other forms of actions that might lead to the front of the knee cruciate ligament (ACL) injury. The results of this study laid the foundation for future research, in addition to finding a causal relationship between kinematics and kinetics of lower limb joints during the landing step of a stop-jump.

\section{CONCLUSION}

The results of this study provided a lot of information for a better understanding of the biomechanical mechanisms during the landing of a stop-jump task between male and female. The hip and knee flexion movement while landing could effectively reduce the impact in landing phase. The hip flexion angle mainly affected longitudinal frictional force during landing. The knee flexion angle of landing not only affected longitudinal frictional force, but also could caused an effective influence on the ground reaction force in the landing phase.

\section{GLOSSARY}

Kinematics, Kinetics, Ground reaction force, Flexion, Torque/Moment, Stop-jump task, Landing

\section{CONFLICT OF INTEREST}

The authors confirm that this article content has no conflict of interest.

\section{ACKNOWLEDGEMENTS}

This study was sponsored by the National Natural Science Foundation of China (81301600), and Outstanding (Postgraduate) Dissertation Growth Foundation of Ningbo University (py2014008).

\section{REFFERENCES}

[1] P. Devita, and WA. Skelly, "Effect of landing stiffness on joint kinetics and energetics in the lower extremity", Med. Sci. Sports Exerc., vol.24, pp. 108-115, 1992.

[2] R.A. Malinzak, S.M. Colby, D.T. Kirkendall, B. Yu, and W.E. Garrett, "A comparison of knee joint motion patterns between men and women in selected athletic tasks", Clin. Biomech., vol. 16, pp. 438-445, 2001.

[3] J.D. Chappell, B. Yu, D.T. Kirkendall, and W.E. Garrett, "A comparison of knee kinetics between male and female recreational athletes in stop-jump tasks", Am. J. Sports Med, vol. 30, pp. 261267, 2002.

[4] J.D. Chappell, B. Yu, D.T. Kirkendall, and W.E. Garrett, "The effect of fatigue on lower extremity kinematics and kinetics during landings of stop-jump tasks", Am. J. Sports Med, vol.33, pp. 10221029, 2005.

[5] M.J. Decker, M.R. Torry, D.J. Wyland, W.I. Sterett, and S.J. Richard, "Gender differences in lower extremity kinematics, kinetics and energy absorption during landing", Clin. Biomech., vol. 18 , pp. $662-669,2003$.

[6] B. Yu, D. Herman, J. Paterson, K. Lu, D.T. Kirkendall, and W.E. Garrett, "The immediate effect of a knee brace with constraint to knee extension on knee kinematics and kinetics during landing of a stop-jump task", Am. J. Sports Med, vol. 32, pp. 1136-1143, 2004.

[7] B.P. Boden, G.S. Dean, J.A. Feagin, and W.E. Garrett, "Mechanisms of ACL Injury", Med. Sci. Sports Exer., vol. 28, pp. 526, 1996.

[8] B. Yu, D.T. Kirkendall, T.N. Taft, and W.E. Garrett, "Lower extremity motor control-related and other risk factors for noncontact anterior cruciate ligament injuries", Instr. Course Lect. vol. 51, pp. 315-324, 2002.

[9] B.C. Fleming, B.D. Beynnon, P.A. Renstrom, R.J. Johnson, C.E. Nichols, G.D. Peura, and B.S. Uh, "The strain behavior of the anterior cruciate ligament during stair climbing: an in vivo study", Arthroscopy, vol. 15, pp. 185-191, 1999.

[10] A. Heijne, B.C. Fleming, P.A. Renstrom, G.D. Peura, B.D Beynnon, and S. Werner, "Strain on the anterior cruciate ligament during closed kinetic chain exercises", Med. Sci. Sports Exerc., vol. 36, pp. 935-941, 2004.

[11] R.M. Nunley, D.W. Wright, J.B. Renner, B. Yu, and W.E. Garrett, "Gender comparison of patella-tendon tibial shaft angle with weight-bearing", Res. Sports Med., vol. 11, pp. 173-185, 2003.

[12] G. DeMorat, P. Weinhold, T. Blackburn, S. Chudik, and W. Garrett, "Aggressive quadriceps loading can induce noncontact anterior cruciate ligament injury", Am. J. Sports Med, vol. 32, pp. 477-483, 2004.

[13] B.C. Fleming, G. Ohlen, P.A. Renstrom, G.D. Peura, B.D. Beynnon, and G.J. Badger, "The effects of compressive load and knee joint torque on peak anterior cruciate ligament strain", Am. $J$. Sports Med, vol. 31, pp. 701-707, 2003.

[14] G. Cerulli, D.L. Benoit, M. Lamontagne, A. Caraffa, and A. Liti, "In vivo anterior cruciate ligament strain behavior during a rapid deceleration movement: case report", Knee Surg. Sports Traumatol. Arthrosc., vol. 11 , pp. 307-311, 2003.

[15] M. Lamontagne, D.L. Benoit, D.K. Ramsey, A. Caraffa, and G. Cerulli, "What can we learn from in vivo biomechanical investigations of lower extremity?" In: Proceedings of XXIII International Symposium of Biomechanics in Sports, pp. 49-56, 2005.

[16] M.N. Samuel, W.R. Hdcomb, and H. Wallmann, "Acute effects of static and ballistic stretching on measures of strength and power", J. Strength Cond. Res., vol. 22, pp. 1422-1428, 2008.

[17] R. Brooke, M. Thomas, E. Dale, and F. Raoul, "Lower extremity bilateral differences during step-close countermovement jumps with concern for gender", J. Strength Cond. Res., vol. 20, p. 608, 2006.

[18] P.S. Sung, and H.S. Park, "Gender differences in ground reaction force following perturbations in subjects with low back pain", Gait Posture, vol. 29, pp. 290-295, 2009.

[19] C.E. Quatman, K.R. Ford, and T.E. Hewett, "Maturation leads to gender differences in landing force and vertical jump performance", Am. J. Sports Med., vol. 5, pp. 806-813, 2006.

[20] E. Pappas, M. Hagins, A. Sheikhzadeh, and D. Rose, "Biomechanical differences between unilateral and bilateral 
landings from a jump: gender differences", Clin. Sports Med., vol. 17, pp. 263-268, 2007.

[21] E.A. Wikstrom, M.D. Tillman, and P.A. Borsa, "Gender and limb differences in dynamic postural stability during landing", Clin. Sports Med., vol. 16, pp. 311-315, 2006.
[22] M.R. Stiffler, J.L. Sanfilippo, and M.A. Brooks, "Counter movement vertical jump force and power differs by gender and sport", Med. Sci. Sports Exerc., vol. 46, pp. 728-729, 2014.

[23] K.B. Shelburne, M.G. Pandy, F.C. Anderson, and M.R. Torry, "Pattern of anterior cruciate ligament force in normal walking", $J$. Biomech. vol. 37, no. 6, pp. 797-805, 2004.

Received: October 17, 2014

Revised: December 30, 2014

Accepted: February 16, 2015

(C) Yin et al.; Licensee Bentham Open.

This is an open access article licensed under the terms of the Creative Commons Attribution Non-Commercial License (http://creativecommons.org/licenses/by-nc/3.0/) which permits unrestricted, non-commercial use, distribution and reproduction in any medium, provided the work is properly cited. 